\title{
PRODUÇÃO DE MUDAS DE ANANDENATHERA MACROCARPA EM DIFERENTES SUBSTRATOS PARA RECUPERAÇÃO DE ÁREAS DEGRADADAS NO CERRADO
}

Kalindy Maressa Soares Monteriro', Patrícia Aparecida de Souza², André Ferreira do Santos ${ }^{2}$, Marcos Vinicius Giongo Alves², Mayanne Alves Pereira ${ }^{3}$

1 Acadêmica de Engenharia Florestal da Universidade Federal do Tocantins (Kalindy14@hotmail.com) Gurupi - Brasil

2 Professor (a) Doutor (a) do Departamento de Engenharia Florestal da Universidade Federal do Tocantins

3 Mestranda em Ciências Florestais da Universidade Federal do Tocantins

Recebido em: 08/09/2015 - Aprovado em: 14/11/2015 - Publicado em: 01/12/2015 DOI: http://dx.doi.org/10.18677/Enciclopedia_Biosfera_2015_213

O cerrado brasileiro apresenta extensas áreas degradadas ou em processo de degradação. Devido à necessidade de recuperação e preservação destas áreas degradadas têm sido levados em consideração para estudos referente a estes impactos as espécies nativas brasileiras do Bioma. Objetivou-se com a realização deste trabalho avaliar o efeito da combinação de diferentes substratos no crescimento de mudas de Anandenathera macrocarpa, para a recuperação de áreas degradadas no cerrado. O experimento foi realizado no Viveiro Florestal da Universidade Federal do Tocantins, delineamento utilizado em DBC, com quatro tratamentos, cinco repetições e uma planta por repetição. Testaram-se as seguintes composições de substratos: T1) Terra de subsolo (testemunha); T2) Terra de subsolo + serragem 1:1 (TS+SR); T3) Terra de subsolo + esterco bovino 1:1 (TS+EB) e T4) Terra de subsolo + serragem + esterco bovino 1:1 (TS+SR+EB). As avaliações foram realizadas aos 30,60,90 e 120 dias após o transplantio. Os indicadores avaliados foram: altura de planta (AP), diâmetro de colo (DC), massa seca aérea (MSA), massa seca radicular (MSR) e massa seca total (MST), e o Índice de Qualidade de Desenvolvimento (IQD). O substrato composto por terra de subsolo mais serragem apresentou o maior desenvolvimento dos parâmetros avaliados, sendo o mais recomendando para a produção de mudas de Anandenathera macrocarpa.

PALAVRAS-CHAVE: bioma, índice de qualidade de Dickson, preservação, substratos orgânicos

\section{PRODUCTION OF ANANDENATHERA MACROCARPA SEEDLINGS GROWN ON DIFFERENT SUBSTRATES FOR RECOVERY OF DEGRADED AREAS IN THE CERRADO.}

\section{ABSTRACT}

The cerrado shows an extensive degraded areas or in the degradation process. Due to the need for restoration and preservation of degraded areas have been taken into 
account in studies concerning these impacts Brazilian native species Biome. The objective of this work was to evaluate the effect of the combination of different substrates on the growth of seedlings Anandenathera macrocarpa for the recovery of degraded areas in the cerrado. The experiment was conducted in the Nursery Forest of the Federal University of Tocantins, design used in DBC, with four treatments, five replications and one plant per repetition. The following substrates compositions were tested: T1) underground land (Whitness); T2) Underground land + sawdust 1: 1 (TS + SR); T3) Underground land + cattle manure 1: 1 (TS + EB) and T4) Underground land + sawdust + cattle manure 1: 1 (TS + SR + EB). The evaluations were performed at 30, 60, 90 and 120 days after transplanting. The indicators evaluated were: plant height $(\mathrm{PH})$, stem diameter (DC), air dry matter (MSA), root dry weight (MSR) and total dry matter (MST), and the Development Quality Index (IQD). The substrate compounded by the subsoil plus sawdust showed the highest development of these parameters, the most recommended for the production of Anandenathera macrocarpa seedlings.

KEYWORDS: Biome, Dickson's quality index, preservation, organic substrates,

\section{INTRODUÇÃO}

O cerrado brasileiro apresenta extensas áreas degradadas ou em processo de degradação. Devido à necessidade de recuperação e preservação destas áreas degradadas têm sido levados em consideração para estudos referente a estes impactos as espécies nativas brasileiras de cada bioma. Sendo necessária a produção de mudas de espécies que possam suprir programas de recuperação e reflorestamentos com espécies nativas, principalmente aquelas que apresentam valor comercial. Segundo CARVALHO FILHO et al. (2003), esse é um dos grandes problemas na recuperação de florestas nativas.

Não há disponibilidade de mudas nativas de qualidade e em quantidade suficiente para suprir a demanda destes programas. Isto ocorre devido a vários fatores, entre estes se citam: a dificuldade de obtenção de sementes, os variados tipos de dormência, o conhecimento limitado sobre a fenologia e a fisiologia de parte significativa das espécies arbustivo-arbóreas dos biomas e investimentos para estes programas (VIANI et al. 2007).

A diversidade encontrada nos biomas brasileiros é grande, com muitas espécies em vários estágios de crescimento e grupos de sucessão. Para escolha das melhores espécies na implantação de mudas no local a ser recuperado e ou preservado realiza-se estudos florísticos como, por exemplo, no Bioma Cerrado. Com efeito, dessa seleção de espécies, para o bioma Cerrado, tem se destacado algumas espécies utilizadas, como: Stryphnodendron Mart, Anadenanthera macrocarpa, Senegalia polyphylla, Myracrodruon urundeuva, Dipteryx alata, Shizolobium amazonicum, entre outras.

A Anandenathera macrocarpa pertence à família Fabaceae-Mimosoideae, apresenta rápido crescimento e é recomendada para reflorestamentos de áreas degradadas. A sua madeira é usada na construção civil, fabricação de móveis, e também usada para carvão e extração de tanino (LORENZI, 2000).

Para a produção das mudas que serão utilizadas na recuperação das áreas degradadas no bioma cerrado, uma das fases mais importantes é a escolha do substrato. O substrato exerce influência marcante na arquitetura do sistema radicular e no estado nutricional das plantas, afetando significativamente a qualidade das mudas que irão para o campo (CARNEIRO, 1983). 
Um substrato é considerado ideal quando o mesmo oferece às mudas condições ótimas para desenvolvimento, como rápido crescimento, sem impedimentos para a raiz, que possibilite circulação de ar, e por último, porém não menos importante, de fácil obtenção e de baixo custo (MELO et al. 2015). Não existe um substrato que seja satisfatório para todas as espécies, por isso é necessário estudos e pesquisas para reconhecimento do melhor substrato para cada uma, com o intuito de obter mudas de qualidade e de baixo preço para recuperar as áreas degradadas.

A necessidade de reverter o quadro atual da degradação ambiental nos biomas brasileiros, principalmente o bioma cerrado, que vem sendo considerado na nova fronteira agrícola do Brasil, gera-se o desafio de se recuperar áreas desmatadas ou degradadas, tendo-se como preocupação ações para o restabelecimento das funções e da estrutura dos ecossistemas respeitando a diversidade de espécies, a sucessão ecológica e a representatividade genética entre populações (BARBOSA, 2000).

Objetivou-se com a realização deste trabalho avaliar o efeito da combinação de diferentes substratos no crescimento de mudas de Anadenanthera macrocarpa, para a recuperação de áreas degradadas no cerrado.

\section{MATERIAL E MÉTODOS}

O experimento foi conduzido no período de setembro de 2013 a outubro de 2014. Na área experimental da Universidade Federal do Tocantins (UFT), Campus Universitário de Gurupi, localizado na região sul do Estado do Tocantins. A altitude da área experimental é de $280 \mathrm{~m}$, e as coordenadas são 1143'45" de latitude e 4904' 07" de longitude.

A classificação climática da região, segundo köppen (1948), é do tipo B1wA'a' úmido com moderada deficiência hídrica. Clima quente e úmido durante todo o ano, a temperatura média anual é de $29,5^{\circ} \mathrm{C}$, com precipi tação anual média de $1804 \mathrm{~mm}$, com um verão chuvoso entre os meses de outubro a abril e um inverno seco entre os meses de maio a setembro.

Foram identificadas árvores matrizes de angico vermelho no Campus Universitário de Gurupi e na região de Gurupi, TO. As árvores matrizes foram marcadas e catalogadas para coleta de sementes para este trabalho e para outros trabalhos que venham ser realizados futuramente.

As sementes coletadas nas árvores matrizes foram beneficiadas no Laboratório de Sementes e colocadas para germinar em sementeira no Viveiro Florestal, após a germinação e quando as plântulas apresentaram 3 a 4 folhas, as mesmas foram transplantadas para os sacos plásticos de $15 \times 25 \mathrm{~cm}$, em viveiro com $50 \%$ de radiação solar.

O delineamento utilizado foi em DBC, com quatro tratamentos, cinco repetições e uma planta por repetição. Os substratos avaliados e suas respectivas proporções encontram-se na tabela 1.

TABELA 1 Tratamentos, substratos e proporção utilizados para a produção de mudas.

\begin{tabular}{llc}
\hline Tratamentos & \multicolumn{1}{c}{ Substratos } & Proporção \\
\hline T1 (TS) & Terra de subsolo (testemunha) & - \\
T2 (TS+SR) & Terra de subsolo + Serragem & $1: 1$ \\
T3 (TS+EB) & Terra de subsolo + Esterco Serragem & $1: 1$ \\
T4 (TS+SR+EB) & Terra de subsolo + Serragem + Esterco de Bovino & $1: 1$ \\
\hline
\end{tabular}


A terra de subsolo e o esterco bovino foram coletados na Universidade Federal do Tocantins; e a serragem foi adquirida em serrarias da cidade.

As avaliações foram realizadas aos 30,60, 90 e 120 dias após o transplantio. Os indicadores avaliados foram: altura de planta (AP), diâmetro de colo (DC), massa seca radicular (MSR), massa seca aérea (MSA) e massa seca total (MST), e Índice de Qualidade de Desenvolvimento (IQD). A altura foi considerada desde o colo até o ápice da planta, medida com régua graduada em centímetros $(\mathrm{cm})$, e o diâmetro do colo medido com paquímetro digital graduado em milímetros $(\mathrm{mm})$, tomado a um centímetro do substrato.

Ao final do experimento para determinação da massa seca, as plantas foram separadas em parte aéreas e raízes. As raízes foram lavadas em água corrente, utilizando-se peneiras de malha fina, para evitar a perda de raízes, principalmente as raízes secundárias. A obtenção das massas foi realizada separadamente acondicionando em sacos de papel devidamente identificados, pesados e colocados em estufa com circulação de ar forçada, à aproximadamente $70 \stackrel{\circ}{\circ}$, durante 72 horas, procedendo à pesagem em balança analítica eletrônica a $(0,01 \mathrm{~g})$.

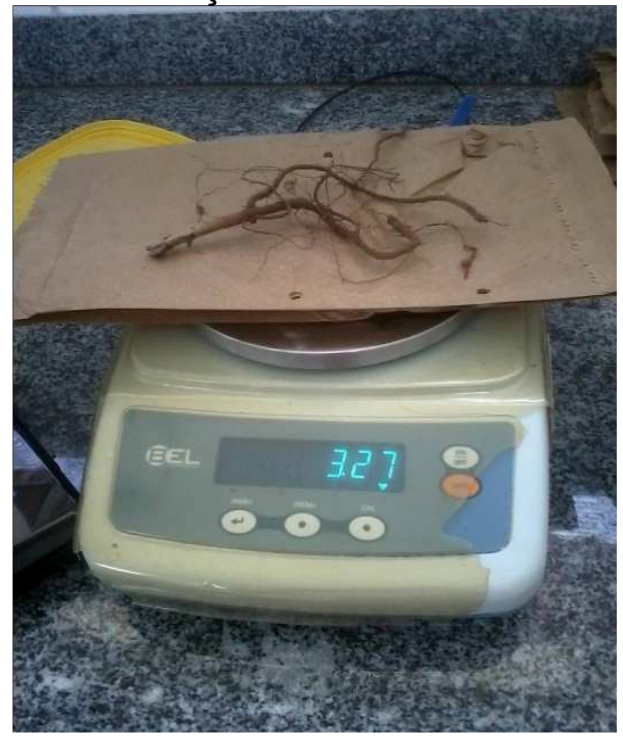

FIGURA 1. Material seco de raiz.

Fonte: os autores.

Para o calculo do índice de qualidade do desenvolvimento (IQD), foi utilizado a metodologia de DICKSOM et al. (1960), considerando os indicadores de massa seca da parte aérea, das raízes e da massa seca total da planta, altura e diâmetro do colo das mudas conforme a equação abaixo.

$$
I Q D=\frac{M S T(\mathrm{~g})}{\frac{H(\mathrm{~cm})}{D C(\mathrm{~cm})}+\frac{\text { PMSPA }(\mathrm{g})}{P M S P R(\mathrm{~g})}}
$$

Onde:

IQD = Índice de desenvolvimento de Dickson, MST = Massa seca total $(\mathrm{g}), \mathrm{H}=$ altura $(\mathrm{cm}), \mathrm{DC}=$ diâmetro do colo $(\mathrm{cm})$, PMSPA = Peso da matéria seca da parte aérea (g) e PMSRA = Peso da matéria seca da raiz $(\mathrm{g})$. 
Os dados obtidos foram tabulados no programa EXCEL e submetidos à análise de variância e teste médias de SCOTT \& KNOTT (1974). Utilizando-se o software SISVAR DEX/UFLA (1999).

\section{RESULTADOS E DISCUSSÃO}

Aos 30 dias após o transplantio o substrato composto por terra de subsolo (testemunha) e o substrato composto por terra de subsolo mais serragem (T2) não diferiram estatisticamente entre si para as variáveis, altura de planta $(A P)$ e diâmetro do colo (DC) (tabela 2). Aos 60, 90, e 120 dias após o transplantio o substrato composto por terra de subsolo mais serragem (T2) proporcionou maior desenvolvimento para a espécie tanto em altura quanto em diâmetro (Tabela 2).

TABELA 2 Altura de plantas (AP) e diâmetro de colo (DC) de mudas de Anadenanthera macrocarpa produzidas em diferentes substratos aos $30,60,90$ e 120 dias após o transplantio.

\begin{tabular}{|c|c|c|c|c|c|c|c|c|}
\hline \multirow[b]{2}{*}{ Tratamentos } & \multicolumn{2}{|c|}{30 DAT } & \multicolumn{2}{|c|}{60 DAT } & \multicolumn{2}{|c|}{90 DAT } & \multicolumn{2}{|c|}{120 DAT } \\
\hline & $\begin{array}{l}\text { AP } \\
(\mathrm{cm})\end{array}$ & $\begin{array}{c}\mathrm{DC} \\
(\mathrm{mm})\end{array}$ & $\begin{array}{l}\text { AP } \\
(\mathrm{cm})\end{array}$ & $\begin{array}{c}\mathrm{DC} \\
(\mathrm{mm})\end{array}$ & $\begin{array}{c}\mathbf{A P} \\
(\mathrm{cm})\end{array}$ & $\begin{array}{r}\mathrm{DC} \\
(\mathrm{mm})\end{array}$ & $\begin{array}{c}\text { AP } \\
(\mathrm{cm})\end{array}$ & $\begin{array}{c}\mathrm{DC} \\
\text { (cm) }\end{array}$ \\
\hline T1 & $6,74 \mathrm{~A}$ & $1,27 \mathrm{~A}$ & $8,50 \mathrm{~B}$ & $1,36 \mathbf{B}$ & 9,41 B & 1,53 B & $11,28 \mathbf{B}$ & $1,65 \mathbf{A}$ \\
\hline T2 & $7,25 \mathbf{A}$ & $1,34 \mathbf{A}$ & $11,86 \mathbf{A}$ & $1,56 \mathbf{A}$ & $14,95 \mathbf{A}$ & $2,17 \mathrm{~A}$ & $21,00 \mathbf{A}$ & $2,38 \mathrm{~A}$ \\
\hline T3 & $4,14 \mathrm{C}$ & $1,16 \mathrm{~B}$ & 5,46 D & $1,16 \mathrm{C}$ & $6,22 \mathrm{~B}$ & $1,27 \mathbf{C}$ & $7,89 \mathrm{~B}$ & $1,34 \mathbf{A}$ \\
\hline T4 & $5,11 \mathrm{~B}$ & $1,20 \mathrm{~B}$ & $6,91 \mathbf{C}$ & $1,21 \mathrm{C}$ & 10,69 B & 1,42 B & 9,52 B & $2,06 \mathrm{~A}$ \\
\hline$\overline{C V}(\%)$ & 18,84 & 12,80 & 23,21 & 11,68 & 70,92 & 15,55 & 38,73 & 82,70 \\
\hline Média & 5,81 & 1,24 & 8,18 & 1,32 & 10,32 & 1,60 & 12,42 & 1,86 \\
\hline
\end{tabular}

Médias seguidas de mesma letra nas colunas não diferem estatisticamente pelo teste de Scott-Knott $(1974)(p \leq 0,05)$.

Anadenanthera macrocarpa faz parte do grupo ecológico das espécies pioneiras, com crescimento rápido a moderado, se desenvolve em solos arenosos, cascalhentos, de baixa fertilidade e também os mais férteis e apresenta preferência por solos bem drenados (LORENZI, 2002). Por ter a capacidade de se desenvolver em solos de baixa fertilidade e bem drenados pode-se explicar o maior desenvolvimento da espécie no substrato composto por terra de subsolo mais serragem (T2), que proporcionou maior desenvolvimento durante o período experimental, quando comparados com os demais. Uma vez que a serragem tem a capacidade de reter a umidade necessária ao desenvolvimento e crescimento do sistema radicular e ao mesmo tempo permite a circulação de ar no substrato.

O trabalho de GONÇALVES et al. (2012) confirmam a baixa exigência da espécie com os resultados encontrados. Os autores concluíram que Anadenanthera macrocarpa tem baixo requerimento nutricional, sendo o nível crítico dos nutrientes no solo e na planta, em geral, menores do que os observados em outras espécies florestais. As mudas aumentam a absorção dos nutrientes $\mathrm{N}, \mathrm{P}, \mathrm{K}, \mathrm{Ca}$ e $\mathrm{Mg}$ à medida que também aumenta o suprimento deles no solo, e em alguns casos não há resposta em crescimento correspondente a esse aumento.

ALVES et al. (2012) avaliando o efeito de diferentes tamanhos de recipientes e de composições de substratos na produção de mudas de Anadenanthera macrocarpa na cidade de Areia estado da Paraíba, no ano de 2011, em condições de viveiro, observaram que não houve diferença estatística entre os substratos a base de solo, areia, esterco e resíduo de caulim. 
CALDEIRA et al. (2008) testando a utilização de diferentes proporções de composto orgânico (serragem de pinus, casca de arroz, resíduo da palmeira-real-daaustrália) nos substratos na cidade de Gaspar/SC no ano de 2008, influenciou significativamente nos parâmetros biométricos e índices de qualidade de mudas de Schinus terebinthifolius. O que foi observado também para Anadenanthera macrocarpa neste trabalho.

Porém SOUZA et al. (2015) avaliando o efeito de diferentes substratos na produção e qualidade de mudas de Eugenia involucrata DC no município de São Gabriel/RS, no ano de 2012, em casa de vegetação, concluíram que o substrato que continha serragem proporcionou um atraso no desenvolvimento de plântulas, em relação aos demais tratamentos. Resultado que corrobaram com os encontrados neste trabalho.

SANT'ANA et al. (2011) estudando o desenvolvimento inicial de angicovermelho submetido a doses de biossólido e composto orgânico na cidade de Alegre/ES, no ano de 2010, em casa de vegetação, concluíram que a adição de composto (esterco animal e folhagens) e de lodo de esgoto ao substrato promoveu ganhos crescentes no desenvolvimento das mudas.

ULIANA et al. (2010) testando a eficiência de diferentes substratos no desenvolvimento de mudas de Cedrela odorata na cidade de Marechal Cândido Rondon - PR, no ano de 2007, observaram que o substrato formado pela mistura de bagaço de cana decomposto com húmus na proporção de 7:3 (v/v) pode ser utilizado como substrato orgânico para a produção de mudas de Anadenanthera macrocarpa. Esse substrato possibilitou maior crescimento em altura, diâmetro do caule e massa seca de raiz.

De acordo com SILVA DO Ó et al. (2015) avaliando o crescimento inicial de angico vermelho em substratos com co-produtos de mineração e matéria orgânica, na cidade de Campina Grande, Patos - PB, em condições de viveiro, observaram que o co-produto de vermiculita associado a estercos caprino e bovino proporcionaram aumento do desenvolvimento nas mudas de angico, com destaque para o esterco caprino.

TABELA 3 Massa seca da parte aérea (MSPA), massa seca de raízes (MSR), relação massa seca de raízes massa seca da parte aérea (MSR/MSPA) e índice de qualidade de Dickson de mudas de Anadenanthera macrocarpa produzidas em diferentes substratos aos 120 dias após o transplantio.

\begin{tabular}{|c|c|c|c|c|}
\hline Tratamentos & MSPA (g) & MSR (g) & MSPA/MSR & IQD \\
\hline $\begin{array}{l}\text { T1 } \\
\text { T2 } \\
\text { T3 } \\
\text { T4 }\end{array}$ & $\begin{array}{l}0,78 \text { B } \\
2,04 \text { A } \\
0,38 \text { C } \\
0,57 \mathbf{C}\end{array}$ & $\begin{array}{l}1,19 \text { B } \\
2,17 \mathbf{A} \\
0,42 \text { C } \\
0,67 \mathbf{C}\end{array}$ & $\begin{array}{l}0,78 \mathbf{A} \\
1,08 \mathbf{A} \\
1,11 \mathbf{A} \\
0,93 \mathbf{A}\end{array}$ & $\begin{array}{l}0,02 \text { B } \\
0,04 \text { A } \\
0,01 \text { B } \\
0,02 \text { B }\end{array}$ \\
\hline $\begin{array}{l}\text { CV (\%) } \\
\text { Média }\end{array}$ & $\begin{array}{c}53,04 \\
0,94\end{array}$ & $\begin{array}{c}64,47 \\
1,11\end{array}$ & $\begin{array}{l}55,61 \\
0,98\end{array}$ & $\begin{array}{c}115,48 \\
0,02\end{array}$ \\
\hline
\end{tabular}

O tratamento composto pelo substrato terra de subsolo mais serragem (T2) proporcionou o maior desenvolvimento em massa seca da parte aérea e massa seca da raiz, as maiores médias foram 2,04 e 2,17, respectivamente, seguido do $T 1$, e depois T3 e T4 que não diferiram estatisticamente entre si. Quanto a relação massa ENCICLOPÉDIA BIOSFERA, Centro Científico Conhecer - Goiânia, v.11 n.22; p.2443 
seca da parte aérea e massa seca da raiz, os tratamentos não diferiram estatisticamente entre si (Tabela 3).

Os resultados encontrados por SOUZA et al. (2015) onde testando o efeito de diferentes substratos na produção de mudas de Anadenanthera macrocarpa, contrariam os resultados do trabalho em questão, pois observaram que o substrato casca de arroz carbonizada quando comparado ao tratamento que continha 0 substrato serragem, apresentou valores médios maiores para todas as variáveis analisadas, ou seja, para massa fresca do sistema radicular, da parte aérea e total, massa seca radicular, da parte aérea e total.

Alves, et al. (2012) testaram o efeito de diferentes tamanhos de recipientes e de composições de substratos na produção de mudas de Anadenanthera macrocarpa, e observaram que não houve efeito dos substratos nas variáveis, massa seca da parte aérea e do sistema radicular.

SCHEER et al. (2012) comparando os dados obtidos para três substratos: substrato comercial à base de casca de Pinus compostada e vermiculita e substratos à base de resíduos de podas de árvores trituradas compostadas com lodo de esgoto aeróbio nas proporções 3:1 (v:v) e 2:1 (v:v), concluíram que os compostos de poda de árvores triturados e lodo de esgoto possuem boas características físico-hídricas e nutricionais, permitindo bons resultados no crescimento (altura, diâmetro, massa seca de folhas e ramos) para mudas de Anadenanthera macrocarpa.

Avaliando as mudas pelo índice de Dickson o substrato composto por terra de subsolo mais serragem apresentou média estatisticamente superior, sendo seu valor 0,04 , enquanto as mudas dos outros tratamentos não diferiram estatisticamente entre si, sendo seus valores T1 e T4 =0,02 e T3 =0,01 (Tabela 3). Segundo BASSACO (2011) o Índice de Qualidade de Dickson (IQD) é um índice bastante robusto, que inclui as relações dos parâmetros morfológicos apresentados, expressando em um único valor a qualidade das mudas, considera-se como valor de referência 0,2. Este índice permite avaliar a qualidade da muda para o campo. Apenas o T3 apresentou média abaixo do valor de referência.

SOUZA et al. (2015) observaram que o índice de qualidade de mudas de Eugenia involucrata não houve diferença significativa para os substratos avaliados, sendo eles: substrato casca de arroz carbonizada; substrato comercial Plantmax®; substrato casca de coco; e substrato serragem.

\section{CONCLUSÕES}

Aos 120 dias após o transplantio de mudas de Anadenanthera macrocarpa, o substrato composto por terra de subsolo e serragem (T2) proporcionou maior desenvolvimento para as variáveis avaliadas, sendo indicado para a produção de mudas desta espécie.

\section{AGRADECIMENTOS}

A Universidade Federal do Tocantins por incentivar a pesquisa.

\section{REFERÊNCIAS}

ALVES, A.S.; OLIVEIRA, L.S.B.; ANDRADE, L.A.; GONÇALVES, G.S.; SILVA, S.M. Produção de mudas de angico em diferentes tamanhos de recipientes e composições de substratos. Revista Verde, Mossoró, v.7, n.2, p.39-44, 2012. 
BARBOSA, L.M. Considerações gerais e modelos de recuperação de formações ciliares. In: RODRIGUES, R.R.; LEITÃO FILHO, H.F. (eds.). Matas Ciliares: Conservação e Recuperação. São Paulo, EDUSP: FAPESP, p. 289-312.2000.

BASSACO, M.V.M. Comportamento fenológico, germinação, produção de mudas e tolerância a saturação hídrica de Sebastiania brasiliensis (Spreng.). Tese (Pósgraduação em Engenharia Florestal) - Universidade Federal do Paraná. $112 \mathrm{p}, 2011$.

CALDEIRA, M.V.W.; ROSA, G.N.; FENILLI, T.A.B.; HARBS, R.M.P.; composto orgânico na produção de mudas de aroeira-vermelha. Revista Scientia Agraria, v. 9, N.1, p.27-33, 2008.

CARNEIRO, J.G.A. Variações na metodologia de produções de mudas florestais afetam os parâmetros morfo-fisiológicos que indicam a sua qualidade. Série Técnica FUPEP, v. 12, p. 1-40, 1983.

CARVALHO FILHO, J.L.S. et al. A. Produção de mudas de (Hymenaea courbaril L.) em diferentes ambientes e composições de substratos. Revista Cerne, Lavras, v. 9, n. 1 , p. $109-118,2003$.

DICKSON, A.; LEAF, A.L.; HOSNER, J.F. Quality appraisal of white spruce and white pine seedling stock in nurseries. Forest Chronicle, v. 36, p.10-13, 1960.

GONÇALVES, E.O.; PAIVA, H.N.; NEVES, J.C.L.; GOMES, J.M. Nutrição de mudas de angico-vermelho (Anadenanthera macrocarpa (BENTH.) BRENAN) submetidas a doses de N, P, K, Ca e Mg. Revista Árvore, Viçosa-MG, v.36, n.2, p.219-228, 2012.

Instituto de Pesquisa Ambiental da Amazônia. Recuperação de Áreas Degradadas. 2010. Disponível em: <http://www.ipam.org.br/saiba-mais/Recuperacao-de-reasDegradadas/5>. Acesso em: 06 Set. 2015

LORENZI, H. Arvores brasileiras: manual de identificação e cultivo de plantas arbóreas do Brasil. 4aㅡ edição. São Paulo: Nova Odesa, 2002. V. 2. 368 p.

MELO, G.W.B.; BORTOLOZZO, A.R.; VARGAS, L. Produção de Morangos no Sistema Semi-Hidropônico. Disponível em: <http://sistemasdeproducao.cnptia.embrapa.br/FontesHTML/Morango/MorangoSemi Hidroponico/substratos.htm >. Acesso: 15 de Out. 2015.

SILVA DO Ó.K.D.; SILVA, G.H.; SANTOS, R.V. Crescimento inicial de angico em substratos com co-produtos de mineração e matéria orgânica. Revista Verde (Pombal - PB - Brasil) v. 10, n.1, p. 178 - 186, jan/mar, 2015.

SOUZA, P.L.T.; VIEIRA, L.R.; BOLIGON, A.A.; VESTENA, S. Produção e qualidade de mudas de Eugenia involucrata DC. em diferentes substratos. Revista Biociências - Universidade de Taubaté, v.21 no 1, 2015. 
SCHEER, M.B.; Carneiro, C.; Bressan, O.A.; Santos, K.G. Compostos de lodo de esgoto para a produção de mudas de Anadenanthera colubrina (Vell.) Brenan. Revista Cerne, v.18, n.4, 613-621. 2012.

ULIANA, M.B.; 2014. Produção de mudas de Anadenanthera macrocarpa em função de substratos alternativos e da frequência de fertirrigação. Revista Floresta, Curitiba, PR, v. 44, n. 2, p. 303 - 312, abr. / jun. 2014.

VIANI, R.A.G.; RODRIGUES, R.R. Sobrevivência em viveiro de mudas de espécies nativas retiradas da regeneração natural de remanescente florestal. Pesquisa Agropecuária Brasileira, v. 42, n.8.2007. 